\title{
Competing endogenous RNA: A novel posttranscriptional regulatory dimension associated with the progression of cancer (Review)
}

\author{
QINGSONG DAI ${ }^{1,2}$, JIXIA LI $^{2}$, KEYUAN ZHOU $^{1}$ and TONG LIANG ${ }^{1}$ \\ ${ }^{1}$ Key Laboratory for Medical Molecule Activity Research; ${ }^{2}$ Department of Biochemistry and Molecular Biology, \\ Guangdong Medical College, Dongguan, Guangdong 523000, P.R. China
}

Received October 2, 2014; Accepted July 7, 2015

DOI: $10.3892 / 01.2015 .3698$

\begin{abstract}
The existence of artificial sponges and antisense oligonucleotides designed to decrease the availability of microRNAs (miRNAs), a family of small non-coding RNAs that target RNA transcripts through miRNA response elements (MREs) involved in gene expression, suggests that miRNAs may also be regulated. The wide range of RNA transcripts harboring MREs, termed competing endogenous RNAs (ceRNAs), includes protein-coding messenger RNAs (mRNAs) and non-coding RNAs, for example long non-coding RNAs, pseudogenes and circular RNAs, which compete for a common pool of miRNAs as natural decoys. These ceRNAs are co-regulated and produce large, complex posttranscriptional regulatory networks, which have been implicated in numerous biological processes. The present review discusses recent discoveries that implicate natural microRNA decoys in the development of cancer.
\end{abstract}

\section{Contents}

1. Introduction

2. Molecular mechanism of ceRNAs and classification

3. ceRNA crosstalk in the progression of cancer

4. Conclusion and perspectives

\section{Introduction}

A single messenger RNA (mRNA) may be regulated by a number of micro RNAs (miRNAs), and a single miRNA is able to regulate multiple mRNAs (1). This finding suggests that the

Correspondence to: Professor Tong Liang, Key Laboratory for Medical Molecule Activity Research, Guangdong Medical College, 1 Xincheng Broadway, Dongguan, Guangdong 523000, P.R. China E-mail: 1t712@126.com

Key words: competing endogenous RNA, microRNA, cancer crosstalk between messenger RNAs and miRNAs is intricate and complex. The canonical role of mRNAs is the delivery of protein-coding information to sites of protein synthesis (2). However, during the past decade, miRNAs, a family of small non-coding RNAs that are important post-transcriptional regulators of gene expression through binding to RNAs, have been well characterized. Numerous researchers have attempted to decipher the crosstalk at this post-transcriptional regulatory stage. The competing endogenous RNA (ceRNA) hypothesis states that all types of RNA transcript compete for miRNAs through a 'language' mediated by miRNA response elements (MREs), and is compelling in this context (3). On the basis of this hypothesis, coding and non-coding RNA transcripts with shared MREs are able to actively communicate with each other to regulate their respective expression levels, thus explaining the consistent expression patterns of ceRNAs and miRNA targets (4).

miRNA inhibitors, termed 'microRNA sponges', are transcripts expressed from certain promoters, which contain multiple, tandem binding sites to for a particular miRNA (5). These artificial sponges may be transiently transfected into cultured cells to derepress miRNA targets, at least as effectively as chemically altered antisense oligonucleotides (6). IPS1, the first endogenous sponge RNA discovered in plants, contains a motif with sequence complementary to miRNA (miR)-399, and functions as an miRNA decoy for sequestering miR-399. This mechanism of inhibition of miRNA activity is termed 'target mimicry', thus resulting in derepression of the miR-399 target PHO2 (7). Artificial target mimics, antisense oligonucleotides and natural miRNA sponges have all been confirmed to compete with miRNA targets for miRNAs, and attenuate the inhibitory activity of miRNAs, indicating the potential ability of non-coding transcripts to interact with miRNAs and control the expression of miRNA target genes at the post-transcriptional regulatory circuit level.

The emerging roles of RNA-RNA crosstalk, as part of a complex posttranscriptional regulatory circuit, have been implicated in human development and disease (Table I). Functional analysis of the miRNA competition and inhibition will likely result in significant insights, regarding basic physiology and disease progression. The present review focused on the molecular mechanisms of ceRNAs and the classification of 
natural RNA species, as well as the implication of ceRNAs in the progression of cancer.

\section{Molecular mechanism of ceRNAs and classification}

mRNAs, transcribed pseudogenes, long non-coding RNAs (lncRNAs) and circular RNAs (circRNAs) that share common MREs with mRNAs may be similarly targeted, sequestering miRNAs to inhibit their interaction with protein-coding mRNAs (Fig. 1A). Therefore, these ceRNAs competing for common miRNAs are also able to regulate each other in ceRNA networks. The strength of this cross-talk is determined by various conditions, including the relative levels of miRNAs and targets, the number of shared miRNA binding sites and the strength of miRNA binding to the target or ceRNA (8). When a given mRNA is upregulated, the repression conferred by its associated targeting miRNAs is decreased, as the total number of MREs exceeds that of the miRNAs themselves (9). Similarly, forced expression of ceRNAs sharing common MREs with protein-coding RNAs sequesters microRNAs and alters the expression levels of miRNA targets (Fig. 1B and C).

mRNA as ceRNAs. It has previously been demonstrated that miRNAs are able to inhibit protein-coding RNAs through binding to MREs of mRNA 3' untranslated regions (3'UTR) (10). The majority of validated ceRNAs are mRNAs, and their ability to compete for miRNA binding and sequester miRNAs from alternate targets may induce biological functions of mRNAs independent of those of their encoded proteins (4). One putative phosphatase and tensin homolog (PTEN) ceRNA, the zinc finger E-box binding homeobox 2 (ZEB2) mRNA, was demonstrated to exert protein-independent and miRNA-dependent regulation of PTEN expression via sequestration of shared miRNAs (11). ZEB2 mRNA has been validated as a PTEN ceRNA and regulates PTEN levels by sequestering at least four miRNAs (miR-181, miR-200b, miR-25 and miR-92a). Attenuation of ZEB2 expression results in the repression of PTEN in human melanomas (11). Another validated PTEN ceRNA is Dickkopf WNT signaling pathway inhibitor $1 \mathrm{mRNA}$ which competes with PTEN mRNA for miR-93 and miR-106a (12). In addition, the 3'UTRs of protein-coding transcripts that typically contain MREs for multiple miRNAs are critical for mRNAs to function as ceRNAs for miRNA targets. The versican (VCAN) 3'UTR has been reported to modulate PTEN levels by sequestering shared miRNAs miR-144 and miR-136 (13). miR-199a-3p and miR-144 target cell cycle regulator retinoblastoma 1 (Rb1), which was also demonstrated to function as a ceRNA for VCAN (13). Thus, the VCAN 3'UTR binds and modulates miRNA activities, acting as a natural miRNA sponge and releasing the Rb1 and PTEN mRNAs for translation. CD34 and FN1 have also been validated as two additional VCAN ceRNAs, which compete for binding to miR-133a, miR-199a-3p, miR-144 and miR-431 (14,15). In light of the ceRNA hypothesis, the 3'UTRs of protein-coding transcripts also likely decoy miRNAs from transcripts with shared MREs in a protein-independent manner, thereby acting as trans regulatory elements to regulating such transcripts (3). Overall, these findings suggest that protein-coding transcripts and 3'UTRs of coding genes may possess significant biological activity through their ability to function as endogenous decoys for miRNAs and thereby regulate miRNA targets.

IncRNAs as ceRNAs. With the ever-expanding number of lncRNAs, increasing numbers of studies have focused on the roles of IncRNAs in epigenetic mechanisms and other biological processes $(16,17)$. Notably, lncRNAs have begun to emerge as natural miRNA decoys, suggesting that they may function as ceRNAs at the post-transcriptional regulatory level (18-20). In particular, the muscle-specific lncRNA LINCMD1 regulates muscle differentiation by binding and sequestering miR-133 and miR-135 (21). Typically, these miRNAs negatively regulate expression of the mastermind-like 1 (MAML1) and myocyte enhancer factor 2C (MEF2C) transcription factors, which induce muscle-specific gene expression. Therefore, by sequestering these miRNAs, LINCMD1 functions as an miRNA decoy and activates MAML1 and MEF2C. In addition, HULC, an lncRNA that has previously been identified to be highly upregulated in liver cancer, acts as an endogenous sponge, downregulating the activity of a series of miRNAs, including miR-372 (22). The inhibition of miR-372 reduces translational repression of its target gene, PRKACB. Another lncRNA, H19, which possesses canonical and non-canonical binding sites for the let-7 family of miRNAs, has been demonstrated to modulate let-7 availability by competing with DICER and HMGA2 as a molecular sponge (23). These studies indicated that lncRNAs may be involved in post-transcriptional regulation by functioning as ceRNAs. Additional lncRNAs functioning as miRNA decoys will be discussed in detail below.

Pseudogenes as ceRNAs. Pseudogenes are defined as genomic loci that resemble functional genes, but are considered biologically inactive as they posses premature stop codons, deletions, insertions and/or frameshift mutations that prevent their effective translation into functional proteins. There are almost as many pseudogenes as there are coding genes, and these pseudogenes represent a significant proportion of the 'transcriptome' (24). Sequencing has revealed that nucleotide sequences contained within pseudogenes are well preserved, suggesting that selective pressure to maintain these genetic elements may exist (25). Processed pseudogenes are generated through retrotransposition and therefore contain no introns; however, they commonly share 5' and 3'UTR sequences with their ancestral genes (26). PTENP1 is supressed by several validated PTEN-targeting miRNAs, and overexpression and RNA interference experiments confirmed that PTENP1 posttranscriptionally regulates the expression of PTEN via shared miRNAs (4). The breast carcinoma amplified sequence 4 (BCAS4) pseudogene, Pbcas4, whose transcripts compete with BCAS4 mRNAs for binding to the common miR-185, is a conserved ceRNA in the mouse and human genome (27). In addition, several other pseudogenes, including the OCT4-pg1, E2F3P1 and CDK4PS, were demonstrated to share binding sites for common miRNAs with their parental genes (4), suggesting that gene regulation by pseudogenes acting as ceRNAs may be a frequent phenomenon. However, to date, few pseudogenes have been functionally characterized, and more evidence supporting this potentially common phenomenon remain to be experimentally validated. 
Table I. Validated non-coding competing endogenous RNAs.

\begin{tabular}{|c|c|c|c|}
\hline ceRNA subtypes & Function & Tissues and species & Reference \\
\hline \multicolumn{4}{|l|}{ Protein-coding RNAs } \\
\hline$Z E B 2$ and $P T E N$ & $\begin{array}{l}\text { Upregulation of PTEN by } \\
\text { sequestering miRNAs }\end{array}$ & Melanoma & $(11)$ \\
\hline$D K K 1$ and $P T E N$ & Modulation of PTEN protein levels & Diabetic cardiomyocytes & (12) \\
\hline$V C A N$ and $P T E N, R b l$ & $\begin{array}{l}\text { Free } R b l \text { and } P T E N \text { mRNAs for } \\
\text { translation, and thus inhibit growth }\end{array}$ & Breast cancer & (13) \\
\hline$V C A N$ and $C D 34, F N 1$ & $\begin{array}{l}\text { VCAN interacts with CD34 and FN1 } \\
\text { as an miRNA decoy }\end{array}$ & Hepatocellular carcinoma & $(14,15)$ \\
\hline$C D 44$ and $C D C 42$ & $\begin{array}{l}\text { Inhibition of cell proliferation, } \\
\text { colony formation, tumor growth }\end{array}$ & $\begin{array}{l}\text { Breast cancer and various } \\
\text { other types of cancer }\end{array}$ & $(37)$ \\
\hline CD44 and Colla1, FN1 & Enhancement of metastasis in vivo & Breast carcinoma & $(57)$ \\
\hline PTEN and CNOT6L, VAPA & $\begin{array}{l}\text { Control downstream PI3K } \\
\text { signaling and cell growth }\end{array}$ & $\begin{array}{l}\text { Glioblastoma and prostate } \\
\text { cancer }\end{array}$ & $(51)$ \\
\hline$H M G A 2$ and $T g f \beta r 3$ & $\begin{array}{l}\text { Competition for let- } 7 \text { occupancy } \\
\text { with the } T G F-\beta \text { co-receptor } T g f \beta r 3\end{array}$ & Lung cancer & $(40)$ \\
\hline \multicolumn{4}{|l|}{ Long non-coding RNAs } \\
\hline LINCMD1 and MAML1, MEF2C & $\begin{array}{l}\text { Control of muscle differentiation } \\
\text { through upregulation of } M A M L 1 \\
\text { and } M E F 2 C \text { transcription factors }\end{array}$ & Mouse and human myoblasts & $(21)$ \\
\hline HULC and PRKACB & $\begin{array}{l}\text { Downregulation of } \\
\text { miRNA-mediated repression }\end{array}$ & Liver cancer & $(22)$ \\
\hline HOTAIR and HER2 & Regulation of HER 2 expression & Gastric cancer & $(47)$ \\
\hline $\begin{array}{l}\text { LincRNA-RoR, OCT4, } \\
\text { SOX2 and NANOG }\end{array}$ & $\begin{array}{l}\text { Mediation of embryonic stem cell } \\
\text { self-renewal and differentiation }\end{array}$ & $\begin{array}{l}\text { Endometrial cancer stem cells, } \\
\text { embryonic stem cells }\end{array}$ & $(33,57)$ \\
\hline IPSI and $P H O 2$ & $\begin{array}{l}\text { Downregulation of } \\
\text { miRNA-mediated repression }\end{array}$ & Arabidopsis thaliana & (7) \\
\hline H19 and DICER, HMGA2 & $\begin{array}{l}\text { Modulation of let- } 7 \text { availability } \\
\text { as a molecular sponge }\end{array}$ & $\begin{array}{l}\text { Mus musculus and } \\
\text { Homo sapiens }\end{array}$ & (23) \\
\hline \multicolumn{4}{|l|}{ Pseudogenes } \\
\hline PTENPI and PTEN & $\begin{array}{l}\text { Upregulation of PTEN } \\
\text { as a ceRNA }\end{array}$ & $\begin{array}{l}\text { Prostate tumor, colon } \\
\text { cancer }\end{array}$ & (4) \\
\hline$K R A S I P$ and $K R A S$ & $\begin{array}{l}\text { Increase } K R A S \text { mRNA } \\
\text { abundance }\end{array}$ & $\begin{array}{l}\text { Prostate cancer, neuroblastoma, } \\
\text { retinoblastoma and } \\
\text { hepatocellular carcimoma }\end{array}$ & (4) \\
\hline Pbcas4 and $B C A S 4$ & $\begin{array}{l}\text { Conserved competitive } \\
\text { endogenous RNA of } B C A S 4\end{array}$ & $\begin{array}{l}\text { Mus musculus and } \\
\text { Homo sapiens }\end{array}$ & $(27)$ \\
\hline \multicolumn{4}{|l|}{ Circular RNAs } \\
\hline $\begin{array}{l}\text { CDRlas or ciRS-7 and } \\
\text { SNCA, EGFR, IRS } 2\end{array}$ & $\begin{array}{l}\text { Downregulation of } \\
\text { miRNA-mediated repression }\end{array}$ & Human and mouse brain & $(29,30)$ \\
\hline
\end{tabular}

ceRNA, competing endogenous RNA; mRNA, messenger RNA; miRNA, micro RNA; PI3K, phosphoinositide 3-kinase; HER2, human epidermal growth factor receptor 2; ciRS-7, circular RNA sponge for miR-7.

circRNA as ceRNAs. circRNAs were initially described in the $1990 \mathrm{~s}$, and the most well-studied circRNA is that generated from the sex-determining Y (SRY) gene, although the biological functions of these RNA circles has remained elusive (28). Recently, SRY, the testis-specific circRNA, was validated as an miR-138 sponge, with ceRNA activity (29), suggesting that these RNA circles may have significant roles in regulatory RNA networks. An antisense transcript to CDR1, termed
CDRlas or circRNA sponge for miR-7, is highly expressed in the human and mouse brain, where circRNA acts as an miR-7 sponge (29,30). CDR1as contains $>70$ selectively conserved miRNA target sites and suppresses miR-7 activity, resulting in the enhanced expression of miR-7 targets (30). circRNAs have been identified in multiple types of tissue, and were demonstrated to be resistant to RNase R treatment (which degrades linear RNA species), and possess longer half-lives compared 


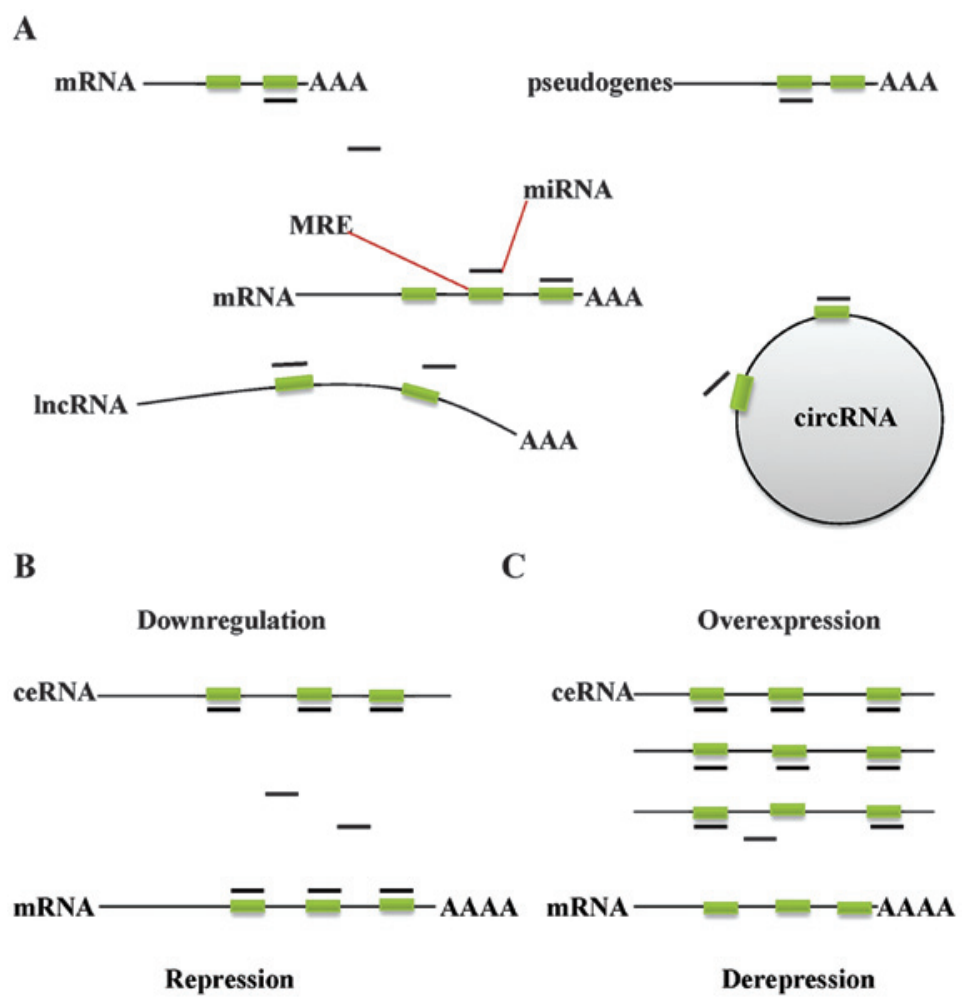

Figure 1. Molecular mechanism of ceRNAs and classification. (A) mRNAs, transcribed pseudogenes, 1 ncRNAs and cirRNAs are able to function as competing endogenous RNAs. (B) Downregulation of ceRNAs results in increased availability of miRNA molecules to bind to mRNA, thereby repressing protein translation. (C) Overexpression of ceRNA results in a reduction in free miRNA abundance, facilitating the derepression of mRNAs that contain identical MREs, thus increasing protein expression levels. ceRNA, competing endogenous RNA; mRNA, messenger RNA; lncRNA, long non-coding RNA; cirRNA, circular RNA; miRNA, micro RNA; MRE, miRNA response element.

with that of their linear RNA transcript counterparts $(31,32)$. Only the linear forms of these RNAs were present in heavy polyribosome fractions, suggesting that the circular forms remain untranslated (31). As a result of their high expression levels and stability, circRNAs with ceRNA activity may be particularly effective modulators of the cross-talk between linear ceRNAs.

\section{3. ceRNA crosstalk in the progression of cancer}

Cross-talk between ceRNAs through shared miRNAs represents a novel layer of gene regulation that may have significant roles in development and disease $(12,21,33)$. In living organisms, although diverse cells that are structured into tissues and organs grow, progress and exert their functions constantly, it is difficult to appreciate the 'perfect mechanism' (34). However, tumorous cells transformed from normal physiological cells that grow beyond their natural boundaries, provide an approach for elucidation of the underlying mechanism. Over several decades of cancer research, six hallmarks of cancer that form the fundamental principle of the process of malignant transformation were proposed and modified (35). These six hallmarks of cancer were: i) sustained proliferative signaling; ii) evasion of growth suppressors; iii) facilitation of replicative immortality; iv) activation of invasion and metastasis; v) induction of angiogenesis; and vi) resistance to cell death. Herein, the present review proposes to introduce ceRNA post-transcriptional regulation into cancer biology and focus on the ceRNA networks involved in these six hallmarks of cancer.
Sustaining proliferative signaling. The capability of constant proliferation is one of the most prominent characteristics of cancer cells. Normal cells tightly regulate the balance of cellular proliferation and death to maintain strict control of cell number, tissue architecture and function. Once this balance is broken, tumor cell signaling cascades that determine their dependence on proliferation signals are deregulated, which results in unlimited growth (36). Ectopic expression of the CD443'UTR binds and inactivates multiple miRNAs, including miR-216a, miR-330 and miR-608, freeing the target mRNA CDC42 from repression (37). CDC42, a gene involved in cell cycle progression, inhibits proliferation, colony formation and tumor growth following enhanced translation. Upregulation of CD44 expression in epithelial ovarian cancer is associated with a favorable outcome and is indicative of enhanced survival time (38). By contrast, the loss of CD44 expression observed in Burkitt's lymphoma, neuroblastoma and prostate cancer was accompanied by oncogenic transformation (39). Thus, it was hypothesized that CD44 mRNA competes with CDC42 for miRNA binding and results in tumorigenesis following the loss of CD44 expression.

In addition to CD44, HMGA2 has been demonstrated to promote lung carcinogenesis via two mechanisms: As a protein-coding gene and as a non-coding RNA (40). HMGA2 is highly expressed in metastatic lung adenocarcinoma, where it has previously been demonstrated to contribute to cancer progression and metastasis (41-43). HMGA2 promotes lung tumor formation by competing with the TGF- $\beta$ co-receptor Tgf $\beta$ r3 for let-7 occupancy, which activates the TGF- $\beta$ 
signaling involved in lung cancer progression. These studies thereby identified a novel gene-expression pathway, where the protein-coding gene, HMGA2, largely behaves independently of its protein-coding function, inducing lung cancer progression as a ceRNA.

The lncRNA, HOTAIR, was initially known for its effects in primary breast tumors and breast cancer metastases, where enhanced HOTAIR expression promoted invasion and metastasis (44). Recent studies also identified upregulated HOTAIR expression in gastric cancer $(45,46)$. HOTAIR functions as a ceRNA, regulating the expression of human epithelial growth factor receptor 2 (HER2) by competing for miR-331-3p. HOTAIR thus functions as an oncogene in gastric pathogenesis by inducing the activation of HER 2 cell signaling networks (47). This finding further supports the hypothesis that the ceRNA function of RNA transcripts is of fundamental significance in oncogenic transformation.

A study revealed that overexpression of the KRAS1P pseudogene 3'UTR resulted in enhanced KRAS mRNA expression and accelerated cell growth (4). Notably, KRAS and KRAS1P transcript levels are co-regulated in prostate cancer, and the KRAS1P locus at 6p11-12 is amplified in various types of human tumor, including retinoblastoma, neuroblastoma and hepatocellular carcinoma (48-50). These findings are indicative of a putative, coding-independent, proto-oncogenic role for KRAS1P, which may be explained by an miRNA decoy mechanism. However, the specific miRNAs involved remain to be elucidated and require further investigation.

Protein-coding RNAs, lncRNAs and pseudogene transcripts are able to function as ceRNAs and contribute to the induction of uncontrolled proliferation. Furthermore, invaluable insight into the function of diverse species may be acquired following analysis of the ceRNA crosstalk involved in cancer progression.

Evading growth suppressors. Several tumor suppressive genes that inhibit cellular growth and proliferation through diverse signaling pathways have been identified, including Tp53, phosphate and tensin homolog (PTEN) and Rb1. The complete loss of a tumor suppressor gene in tumor cells represents one mechanism of achieving constant proliferative capability, for example Tp53. However, another mechanism is also used to posttranscriptionally modulate and inactivate the tumor suppressor genes.

The pseudogene PTENP1 competes with PTEN for miRNA binding, thereby modulating the derepression of specific miRNA targets (4). Following the loss of PTENP1, the decreased translation of PTEN was unable to exert tumor suppressive functions by inhibiting the phosphoinositide 3-kinase (PI3K)/AKT signaling pathway. The existence of genomic copy number losses at the PTENP1 locus supports the hypothesis that PTENP1 is a tumor suppressor gene, and may be under selective pressure to undergo copy number losses in cancer. Another two protein-coding RNAs, CNOT6L and (vesicle-associated membrane protein)-associated protein A (VAPA), appeared to phenocopy PTEN loss-mediated AKT activation based on their function as PTEN ceRNAs (51). Thus, silencing of the tumor suppressor gene PTEN due to loss of CNOT6L and VAPA, resulted in evasion of growth inhibition. The identification of significant copy number losses of the
VAPA and CNOT6L genomic loci supported the hypothesis that these protein-coding RNAs exert tumor-suppressive effects.

The ZEB2 protein has previously been established as an activator of the epithelial-to-mesenchymal transition (EMT) and was demonstrated to be involved in the promotion of cancer progression and metastasis in certain instances of epithelial cancer $(52,53)$. However, the ZEB2 transcript functions as a PTEN ceRNA and modulates PTEN protein levels in an miRNA-dependent, protein coding-independent manner (11). ZEB2 and PTEN are co-regulated, and ZEB2 levels are commonly attenuated in human cancers. PTEN antagonizes PI3K/AKT signaling, therefore attenuation of ZEB2 expression activates the PI3K/AKT pathway, enhancing cell transformation.

Expression of the VCAN 3'UTR has been reported to bind and modulate miRNA activities, releasing PTEN mRNAs for translation, and resulting in reduced cell proliferation and tumor growth (13). In addition to its role as a PTEN ceRNA, VCAN was also demonstrated to act as a ceRNA for the cell-cycle regulator Rb1 by competing for common miRNAs, upregulating this crucial tumor suppressor in vitro and in vivo (13). Expression of Rb1 and PTEN were synergistically upregulated in vitro and in vivo, suggesting that VCAN transcripts acting as ceRNAs contribute to tumorigenesis via the $\mathrm{Rb} 1$ and $\mathrm{PI} 3 \mathrm{~K}$ signaling pathways.

In addition, an lncRNA tumor suppressor, PTCSC3, was found to be downregulated in thyroid cancer cells (54). PTCSC3 functions as a ceRNA by sequestering miR-574-5p, thereby inducing cell cycle arrest. However, further investigations regarding the miRNA targets co-regulated with PTCSC3 in thyroid cancer is required.

Collectively, protein-coding RNAs and noncoding RNAs, including pseudogene transcripts and lncRNAs, attenuate proliferation inhibition and promote tumorigenesis through the sequestration of miRNAs as ceRNAs.

Enabling replicative immortality. In contrast to normal cells that are only able to complete a limited number of cell divisions, tumor cells extensively self-renew, similarly to embryonic stem cells (ESCs). Thus, it was hypothesized that tumor cells acquire this ESC-like property by reprogramming normal cells and enabling replicative immortality. A large intergenic noncoding RNA, linc-RoR, whose expression is linked to pluripotency and self-renewal, has been identified as a key reprogramming regulator (55). Linc-RoR functions as a key ceRNA, linking the network of miRNAs and core transcription factors (TFs), including Oct4, Sox 2 and Nanog, and protecting these core TFs from miRNA-mediated suppression in self-renewing human ESCs (56). Linc-RoR also functions as a 'sponge' against mediation of the differentiation of endometrial cancer stem cells by miR-145, suggesting that endogenous linc-RoR may have a key role in cancer stem cell (CSC) maintenance and pluripotency (33). The identification of additional ceRNAs associated with self-renewal of CSCs requires further exploration.

Activating invasion and metastasis. Invasion and distant metastases occur in diverse types of advanced tumor. Notably, the majority of patients with cancer succumb to these 
metastases rather than the primary tumor. Frequently, cancer cells undergo morphological changes and alter their cell-cell or cell-matrix interactions. Altered expression of components of the extracellular matrix (ECM) has a significant role in cancer metastasis. It was previously revealed that overexpression of the CD44 3'UTR resulted in enhanced cell motility, invasion and adhesion in the MDA-MB-231 human breast carcinoma cell line, and also enhanced metastasis in vivo (57). miRNAs which interact with the CD44 3'UTR also have binding sites in other matrix-encoding mRNA 3'UTRs, including collagen type $1 \alpha 1$ (Coll $\alpha 1)$, which is suppressed by miR-328, as well as fibronectin type 1 (FN1), which is repressed by miR-512-3p, miR-491 and miR-671 (57). The involvement of FN1 in cancer cell migration and metastasis has also been well documented (58). Col1 $\alpha 1$ is a major ECM component, which affects cell behavior and maintains tissue architecture. Protein analysis demonstrated that, following transfection of the CD44 3'UTR, the expression levels of CD44, Coll 11 and FN1 were synergistically upregulated in vitro and in vivo (57). Therefore, the non-coding 3'UTR of CD44 interacts with numerous miRNAs that target ECM properties and activate invasion and metastasis. Furthermore, overexpression of the VCAN 3'UTR would compete with FN1 for binding to three miRNAs, miR-133a, miR-431 and miR-199a*, resulting in ectopic invasion and metastasis in human hepatocellular carcinoma $(14,15)$.

In addition, Coll $\alpha 2$ was confirmed to be one of the ceRNA targets of HMGA2, which also competes for let-7 binding (44). Let-7 targets Coll $\alpha 2$ and inhibits cell migration in hepatocellular carcinoma (59). These findings suggest that HMGA2 may also function as a ceRNA and promote invasion and metastasis. These invasive and migratory phenotypic consequences remain to be validated following silencing of HMGA2 or overexpression of HMGA2 3'UTR.

Inducing angiogenesis. Angiogenesis, a process which is typically confined to embryonic development, may be reactivated under certain conditions in patients with tumors. The secreted protein, vascular endothelial growth factor-A (VEGFA), whose expression may be induced by hypoxia or oncogenic signals, is an activator of angiogenesis (60). Analysis of gene expression in glioblastoma, in combination with matched miRNA profiles, revealed posttranscriptional regulation of notable magnitude, comprising $>248,000$ miRNA-mediated interactions (61). In particular, ectopic expression of PTEN or Rb1 3'UTRs induced a 1.5-fold upregulation of VEGFA, in an miRNA-dependent manner. These data indicated that VEGFA functioned as natural miRNA sponge and was involved in the posttranscriptional regulation of gene expression. Therefore, the determination of whether overexpression of PTEN or Rb1 3'UTRs promotes angiogenesis by competing for miRNA binding with VEGFA in glioblastoma requires experimental validation.

Resisting cell death. Three major pathways, apoptosis, autophagy and necrosis result in cell death, and highly malignant types of cancer are able to attenuate cell death and become therapy resistant. In cancer, the role of miRNA in drug resistance has been well studied over the last few years $(62,63)$. ceRNAs which compete for miRNA binding with RNA transcripts involved in these three pathways remain to be researched.

\section{Conclusion and perspectives}

The emerging ceRNA hypothesis is a novel field of RNA biology. Studies by several groups have revealed that ceRNAs function as posttranscriptional regulators of gene expression by decoying miRNAs from other RNA transcripts $(4,11,21)$. Protein-coding RNAs, pseudogenes, lncRNAs and circRNAs serve as natural miRNA sponges through sequestration of miRNAs. Previously, loss of CD44 expression reduced CDC42 as miRNA decoys and promoted proliferation. In addition, CD44 competed with FN1 and Coll $\alpha 1$ as a ceRNA leading to metastasis in human tumors $(37,57)$. Expression of VCAN 3'UTRs reduced tumor growth by competing with Rb1 and PTEN mRNAs, and contributed to invasion by serving as an FN1 ceRNA (13-15). These findings suggest that tumorigenesis, accompanied by metastasis and other hallmarks of cancer, may be partially attributed to ceRNAs, which compete with diverse RNA transcripts for various miRNAs. Furthermore, an equilibrium exists between ceRNAs and miRNAs in the post-transcriptional regulatory network, which when perturbed, contributes to disease pathogenesis.

Although the ceRNA field remains in its infancy, the rapid discovery of ceRNA-associated miRNAs and miRNA targets has contributed to the development of miRNA-based therapeutics (64). However, certain questions need to be addressed. The developing progress of antisense or small interfering RNA drugs has been limited by stability, specificity and delivery problems. In addition, since a single miRNA is able to recognize and inhibit a large number of target genes, altering a single miRNA may affect multiple, unintended genes. Conversely, a single gene may be targeted by numerous miRNAs, and whether varying one miRNA is sufficient to affect a specific gene target remains to be elucidated (65). The ceRNA mechanism provides a novel perspective to gene therapy for cancer. ceRNAs are natural miRNA sponges and may possess improved biological stability compared with that of miRNAs, particularly circRNAs $(31,32)$. In addition, ceRNAs combine and sequester multiple miRNAs, and these common miRNAs ensure that the specific ceRNA target harboring the common MREs will be largely affected. Furthermore, off-target effects are significantly reduced. Taking into consideration the undesired gene targets altered by miRNA transduction, the expression of mRNA-associated ceRNAs may be restored to physiological levels by ceRNAs, so that miRNAs and mRNAs will be balanced. Extensive studies of miRNAs and ceRNAs are required, as these may serve as therapeutic targets for the treatment of cancer to enhance sensitivity and attenuate drug resistance.

\section{References}

1. Friedman RC, Farh KK, Burge CB and Bartel DP: Most mammalian mRNAs are conserved targets of microRNAs. Genome Res 19: 92-105, 2009.

2. Katahira J: Nuclear export of messenger RNA. Genes (Basel) 6: 163-184, 2015.

3. Salmena L, Poliseno L, Tay Y, Kats L and Pandolfi PP: A ceRNA hypothesis: The Rosetta Stone of a hidden RNA language? Cell 146: 353-358, 2011. 
4. Poliseno L, Salmena L, Zhang J, Carver B, Haveman WJ and Pandolfi PP: A coding-independent function of gene and pseudogene mRNAs regulates tumour biology. Nature 465: 1033-1038, 2010

5. Ebert MS, Neilson JR and Sharp PA: MicroRNA sponges: Competitive inhibitors of small RNAs in mammalian cells. Nat Methods 4: 721-726, 2007.

6. Ebert MS and Sharp PA: Emerging roles for natural microRNA sponges. Curr Biol 20: R858-R861, 2010.

7. Franco-Zorrilla JM, Valli A, Todesco M, Mateos I, Puga MI, Rubio-Somoza I, Leyva A, Weigel D, García JA and Paz-Ares J: Target mimicry provides a new mechanism for regulation of microRNA activity. Nat Genet 39: 1033-1037, 2007.

8. Ala U, Karreth FA, Bosia C, Pagnani A, Taulli R, Léopold V, Tay Y, Provero P, Zecchina R and Pandolfi PP: Integrated transcriptional and competitive endogenous RNA networks are cross-regulated in permissive molecular environments. Proc Natl Acad Sci USA 110: 7154-7159, 2013.

9. Arvey A, Larsson E, Sander C, Leslie CS and Marks DS: Target mRNA abundance dilutes microRNA and siRNA activity. Mol Syst Biol 6: 363, 2010.

10. Bartel DP: MicroRNAs: Target recognition and regulatory functions. Cell 136: 215-233, 2009

11. Karreth FA, Tay Y, Perna D, Ala U, Tan SM, Rust AG DeNicola G, Webster KA, Weiss D, Perez-Mancera PA, et al: In vivo identification of tumor-suppressive PTEN ceRNAs in an oncogenic BRAF-induced mouse model of melanoma. Cell 147: 382-395, 2011

12. Ling S, Birnbaum Y, Nanhwan MK, Thomas B, Bajaj M, Li Y Li Y and Ye Y: Dickkopf-1 (DKK1) phosphatase and tensin homolog on chromosome 10 (PTEN) crosstalk via microRNA interference in the diabetic heart. Basic Res Cardiol 108: 352, 2013.

13. Lee DY, Jeyapalan Z, Fang L, Yang J, Zhang Y, Yee AY, Li M, Du WW, Shatseva T and Yang BB: Expression of versican 3'-untranslated region modulates endogenous microRNA functions. PLoS One 5: e13599, 2010.

14. Lee DY, Shatseva T, Jeyapalan Z, Du WW, Deng Z and Yang BB: A 3'-untranslated region (3'UTR) induces organ adhesion by regulating miR-199a* functions. PLoS One 4: e4527, 2009.

15. Fang L, Du WW, Yang X, Chen K, Ghanekar A, Levy G, Yang W, Yee AJ, Lu WY, Xuan JW, et al: Versican 3'-untranslated region (3'-UTR) functions as a ceRNA in inducing the development of hepatocellular carcinoma by regulating miRNA activity. FASEB J 27: 907-919, 2013

16. Nagano $T$ and Fraser P: No-nonsense functions for long noncoding RNAs. Cell 145: 178-181, 2011.

17. Mercer TR and Mattick JS: Structure and function of long noncoding RNAs in epigenetic regulation. Nat Struct Mol Biol 20: 300-307, 2013.

18. Griffiths-Jones S, Saini HK, van Dongen S and Enright AJ: miRBase: Tools for microRNA genomics. Nucleic Acids Res 36 D154-D158, 2008.

19. Paraskevopoulou MD, Georgakilas G, Kostoulas N, Reczko M, Maragkakis M, Dalamagas TM and Hatzigeorgiou AG: DIANA-LncBase: Experimentally verified and computationally predicted microRNA targets on long non-coding RNAs. Nucleic Acids Res 41: D239-D245, 2013.

20. Chiyomaru T, Fukuhara S, Saini S, Majid S, Deng G Shahryari V, Chang I, Tanaka Y, Enokida H, Nakagawa M, et al: Long non-coding RNA HOTAIR is targeted and regulated by miR-141 in human cancer cells. J Biol Chem 289: 12550-12565, 2014

21. Cesana M, Cacchiarelli D, Legnini I, Santini T, Sthandier O, Chinappi M, Tramontano A and Bozzoni I: A long noncoding RNA controls muscle differentiation by functioning as a competing endogenous RNA. Cell 147: 358-369, 2011.

22. Wang J, Liu X, Wu H, Ni P, Gu Z, Qiao Y, Chen N, Sun F and Fan Q: CREB up-regulates long non-coding RNA, HULC expression through interaction with microRNA-372 in liver cancer. Nucleic Acids Res 38: 5366-5383, 2010.

23. Kallen AN, Zhou XB, Xu J, et al: The imprinted H19 lncRNA antagonizes Let-7 microRNAs. Mol Cell 52: 101-112, 2013.

24. Harrison PM, Zheng D, Zhang Z, Carriero N and Gerstein M: Transcribed processed pseudogenes in the human genome: An intermediate form of expressed retrosequence lacking protein-coding ability. Nucleic Acids Res 33: 2374-2383, 2005.

25. Pink RC, Wicks K, Caley DP, Punch EK, Jacobs L and Carter DR Pseudogenes: Pseudo-functional or key regulators in health and disease? RNA 17: 792-798, 2011.
26. D'Errico I, Gadaleta G and Saccone C: Pseudogenes in metazoa: Origin and features. Brief Funct Genomic Proteomic 3: 157-167, 2004.

27. Marques AC, Tan J, Lee S, Kong L, Heger A and Ponting CP: Evidence for conserved post-transcriptional roles of unitary pseudogenes and for frequent bifunctionality of mRNAs. Genome Biol 13: R102, 2012

28. Capel B, Swain A, Nicolis S, Hacker A, Walter M, Koopman P, Goodfellow P and Lovell-Badge R: Circular transcripts of the testis-determining gene Sry in adult mouse testis. Cell 73: 1019-1030, 1993

29. Hansen TB, Jensen TI, Clausen BH, Bramsen JB, Finsen B, Damgaard CK and Kjems J: Natural RNA circles function as efficient microRNA sponges. Nature 495: 384-388, 2013.

30. Memczak S, Jens M, Elefsinioti A, Torti F, Krueger J, Rybak A, Maier L, Mackowiak SD, Gregersen LH, Munschauer M, et al: Circular RNAs are a large class of animal RNAs with regulatory potency. Nature 495: 333-338, 2013.

31. Jeck WR, Sorrentino JA, Wang K, Slevin MK, Burd CE, Liu J, Marzluff WF and Sharpless NE: Circular RNAs are abundant, conserved and associated with ALU repeats. RNA 19: 141-157, 2013.

32. Salzman J, Gawad C, Wang PL, Lacayo N and Brown PO Circular RNAs are the predominant transcript isoform from hundreds of human genes in diverse cell types. PLoS One 7: e30733, 2012

33. Zhou X, Gao Q, Wang J, Zhang X, Liu K and Duan Z: Linc-RNA-RoR acts as a 'sponge' against mediation of the differentiation of endometrial cancer stem cells by microRNA-145 Gynecol Oncol 133: 333-339, 2014.

34. Slaidina $\mathrm{M}$ and Lehmann R: Translational control in germline stem cell Development. J Cell Biol 207: 13-21, 2014.

35. Gutschner T and Diederichs S: The hallmarks of cancer: A long non-coding RNA point of view. RNA Biol 9: 703-719, 2012

36. Hanahan D and Weinberg RA: Hallmarks of cancer: The next generation. Cell 144: 646-674, 2011.

37. Jeyapalan Z, Deng Z, Shatseva T, Fang L, He C and Yang BB: Expression of CD44 3'-untranslated region regulates endogenous microRNA functions in tumorigenesis and angiogenesis. Nucleic Acids Res 39: 3026-3041, 2011.

38. Voutilainen K, Anttila M, Sillanpää S, Tammi R, Tammi M, Saarikoski S and Kosma VM: Versican in epithelial ovarian cancer: Relation to hyaluronan, clinicopathologic factors and prognosis. Int J Cancer 107: 359-364, 2003.

39. Herrlich P, Morrison H, Sleeman J, Orian-Rousseau V, König H, Weg-Remers S and Ponta H: CD44 acts both as a growth- and invasiveness-promoting molecule and as a tumor-suppressing cofactor. Ann NY Acad Sci 910: 106-118, 2000.

40. Kumar MS, Armenteros-Monterroso E, East P, Chakravorty P, Matthews N, Winslow MM and Downward J: HMGA2 functions as a competing endogenous RNA to promote lung cancer progression. Nature 505: 212-217, 2014.

41. Di Cello F, Hillion J, Hristov A, Wood LJ, Mukherjee M, Schuldenfrei A, Kowalski J, Bhattacharya R, Ashfaq R and Resar LM: HMGA2 participates in transformation in human lung cancer. Mol Cancer Res 6: 743-750, 2008.

42. Meyer B, Loeschke S, Schultze A, Weigel T, Sandkamp M, Goldmann T, Vollmer E and Bullerdiek J: HMGA2 overexpression in non-small cell lung cancer. Mol Carcinog 46: 503-511, 2007.

43. Winslow MM, Dayton TL, Verhaak RG, Kim-Kiselak C, Snyder EL, Feldser DM, Hubbard DD, DuPage MJ, Whittaker CA, Hoersch S, et al: Suppression of lung adenocarcinoma progression by Nkx2-1. Nature 473: 101-104, 2011

44. Gupta RA, Shah N, Wang KC, Kim J, Horlings HM, Wong DJ, Tsai MC, Hung T, Argani P, Rinn JL, et al: Long non-coding RNA HOTAIR reprograms chromatin state to promote cancer metastasis. Nature 464: 1071-1076, 2010.

45. Endo H, Shiroki T, Nakagawa T, Yokoyama M, Tamai K, Yamanami H, Fujiya T, Sato I, Yamaguchi K, Tanaka N, et al: Enhanced expression of long non-coding RNA HOTAIR is associated with the development of gastric cancer. PLoS One 8: e77070, 2013

46. Hajjari M, Behmanesh M, Sadeghizadeh M and Zeinoddini M: Up-regulation of HOTAIR long non-coding RNA in human gastric adenocarcinoma tissues. Med Oncol 30: 670, 2013.

47. Liu XH, Sun M, Nie FQ, Ge YB, Zhang EB, Yin DD, Kong R, Xia R, Lu KH, Li JH, et al: Lnc RNA HOTAIR functions as a competing endogenous RNA to regulate HER 2 expression by sponging miR-331-3p in gastric cancer. Mol Cancer 13: 92, 2014. 
48. van der Wal JE, Hermsen MA, Gille HJ, Schouten-Van Meeteren NY, Moll AC, Imhof SM, Meijer GA, Baak JP and van der Valk P: Comparative genomic hybridisation divides retinoblastomas into a high and a low level chromosomal instability group. J Clin Pathol 56: 26-30, 2003.

49. Zimonjic DB, Keck CL, Thorgeirsson SS and Popescu NC: Novel recurrent genetic imbalances in human hepatocellular carcinoma cell lines identified by comparative genomic hybridization. Hepatology 29: 1208-1214, 1999.

50. Plantaz D, Mohapatra G, Matthay KK, Pellarin M, Seeger RC and Feuerstein BG: Gain of chromosome 17 is the most frequent abnormality detected in neuroblastoma by comparative genomic hybridization. Am J Pathol 150: 81-89, 1997.

51. Tay Y, Kats L, Salmena L, Weiss D, Tan SM, Ala U, Karreth F, Poliseno L, Provero P, Di Cunto F, et al: Coding-independent regulation of the tumor suppressor PTEN by competing endogenous mRNAs. Cell 147: 344-357, 2011.

52. Gregory PA, Bert AG, Paterson EL, Barry SC, Tsykin A, Farshid G, Vadas MA, Khew-Goodall Y and Goodall GJ: The miR-200 family and miR-205 regulate epithelial to mesenchymal transition by targeting ZEB1 and SIP1. Nat Cell Biol 10: 593-601, 2008.

53. Vandewalle C, Comijn J, De Craene B, Vermassen P, Bruyneel E, Andersen H, Tulchinsky E, Van Roy F and Berx G: SIP1/ZEB2 induces EMT by repressing genes of different epithelial cell-cell junctions. Nucleic Acids Res 33: 6566-6578, 2005.

54. Fan M, Li X, Jiang W, Huang Y, Li J and Wang Z: A long non-coding RNA, PTCSC3, as a tumor suppressor and a target of miRNAs in thyroid cancer cells. Exp Ther Med 5: 1143-1146, 2013.

55. Loewer S, Cabili MN, Guttman M, Loh YH, Thomas K, Park IH, Garber M, Curran M, Onder T, Agarwal S, et al: Large intergenic non-coding RNA-RoR modulates reprogramming of human induced pluripotent stem cells. Nat Genet 42: 1113-1117, 2010.
56. Wang Y, Xu Z, Jiang J, Xu C, Kang J, Xiao L, Wu M, Xiong J, Guo $\mathrm{X}$ and Liu H: Endogenous miRNA sponge lincRNA-RoR regulates Oct4, Nanog and Sox 2 in human embryonic stem cell self-renewal. Dev Cell 25: 69-80, 2013.

57. Rutnam ZJ and Yang BB: The non-coding 3'UTR of CD44 induces metastasis by regulating extracellular matrix functions. J Cell Sci 125: 2075-2085, 2012.

58. Waalkes S, Atschekzei F, Kramer MW, Hennenlotter J, Vetter G, Becker JU, Stenzl A, Merseburger AS, Schrader AJ, Kuczyk MA and Serth J: Fibronectin 1 mRNA expression correlates with advanced disease in renal cancer. BMC Cancer 10: 503, 2010.

59. Ji J, Zhao L, Budhu A, Forgues M, Jia HL, Qin LX, Ye QH, Yu J, Shi X, Tang ZY and Wang XW: Let-7g targets collagen type I alpha 2 and inhibits cell migration in hepatocellular carcinoma. $\mathrm{J}$ Hepatol 52: 690-697, 2010.

60. Ferrara N: Vascular endothelial growth factor. Arterioscler Thromb Vasc Biol 29: 789-791, 2009.

61. Sumazin P, Yang X, Chiu HS, Chung WJ, Iyer A, Llobet-Navas D, Rajbhandari P, Bansal M, Guarnieri P, Silva J and Califano A: An extensive microRNA-mediated network of RNA-RNA interactions regulates established oncogenic pathways in glioblastoma. Cell 147: 370-381, 2011.

62. Giovannetti E, Erozenci A, Smit J, Danesi R and Peters GJ: Molecular mechanisms underlying the role of microRNAs (miRNAs) in anticancer drug resistance and implications for clinical practice. Crit Rev Oncol Hematol 81: 103-122, 2012.

63. Allen KE and Weiss GJ: Resistance may not be futile: MicroRNA biomarkers for chemoresistance and potential therapeutics. Mol Cancer Ther 9: 3126-3136, 2010.

64. Cho WC: MicroRNAs: Potential biomarkers for cancer diagnosis, prognosis and targets for therapy. Int J Biochem Cell Biol 42: 1273-1281, 2010

65. Cho WC: MicroRNAs in cancer-from research to therapy. Biochim Biophys Acta 1805: 209-217, 2010. 